\title{
STRAIN GRADIENT CONTINUUM MODELS FOR LINEAR PANTOGRAPHIC STRUCTURES: A CLASSIFICATION BASED ON MATERIAL SYMMETRIES
}

\author{
YOSRA RAHALI, JEAN-FRANÇOIS GANGHOFFER, FREJ CHAOUACHI \\ AND ALI ZGHAL
}

Communicated by Andrei Ludu

\begin{abstract}
We analyze the mechanics of planar networks of extensible fibers, for which we derive the general form of the mechanical energy. We consider especially networks made of two sets of non orthogonal and non equivalent fibers, called the parallelogram structure, with variants obtained as specific patterns called the square, rectangular, and rhombic structures. The fibers of the network are assumed to obey Bernoulli kinematics. A second order gradient continuum is obtained. The arguments of the energy of these four patterns are obtained based on the material symmetry group of the considered structures.
\end{abstract}

MSC: 70 XX, 70 CXX, 70 G65, 74 BXX, 74 GXX, 74 QXX

Keywords: Determination of effective properties, elastic materials, equilibrium (steady-state) problems, homogenization

\section{Introduction}

Network materials made of a repetitive set of beams continue to attract the interest of many researchers (see for instance $[4,14,16]$ ), due to their low weight and interesting mechanical performances in comparison to bulk materials. The relationship between the material microstructure and the resulting properties is the key to optimization and design of lightweight, strong, and tough materials and structures [7]. Examples include artificial textile materials, soft biological structures like skin or the arterial wall, for which open problems in this field are related to the development of suitable constitutive models accounting for the anisotropy induced by the fibers, and the presence of large strains. Despite the availability of powerful machines, it may be cumbersome to compute the mechanical response of such discrete structures, thus there is a need for suitable continuum constitutive models that can be used at the macroscale for the purpose of structural computations.

The objective of this work is the derivation of the energy of a set of two inequivalent fibers, a situation representative of a planar network of extensible and flexible 


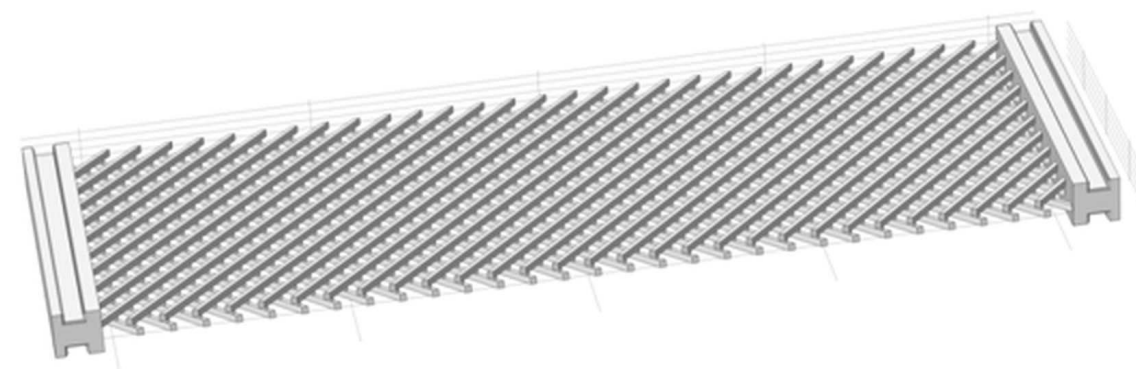

Figure 1. Pantographic structure [13].

fibers. As will become clear later on, the effective energy shall involve both first and second order gradients of the displacement field, thereby pointing the need to consider the class of second order gradient continua as a proper modeling framework for such networks.

Several authors have turned their attention towards the development of rigorous homogenization procedures showing the ability to account for the heterogeneous nature of the material at the micro-level using a second gradient macroscopic constitutive law [2,9]. Works in this direction consider both linear elastic materials [8] and non linear materials $[1,6]$. Higher order homogenization schemes have been built for architectured materials in both the linear and nonlinear regimes in the work of Trinh [17].

Especially, considering network materials, the pantograph is well-known, for it is a typical example of a structure exhibiting no first order elasticity, but instead its elasticity appears directly at second order when it is viewed as a continuum, thus it is a second order grade continuum [13]. It is made of two equivalent inextensible beams connected at right angle by a pivot (Fig. 1).

The inextensibility of the fibers raises specific issues from both theoretical and numerical viewpoint, as exposed into details in [13]. The most popular theories developed in the literature for the mechanical analysis started in 1955 from the works of Rivlin [15], who proposed a theory of planar networks made of inextensible cords. This was followed by a series of works of Pipkin [10-12], who further developed the theory of inextensible cords. In the same period, Wang and Pipkin $[18,19]$ proposed a model of inextensible fibers with bending stiffness, which appears as a special case of the finite-deformation plate theory. This theory was then extended by Luo and Steigmann [5] who considered both bending and twisting effects for 3D configurations.

Pantographic structures are constituted by two families of beams, called also fibers, connected by an internal pivot which allow a relative rotation. 
We shall in the present work analyze the mechanics of more general pantographic structures made of two extensible, non orthogonal and non equivalent fibers.

As to notations, vectors and second order tensors are denoted with boldface symbols.

\section{General Form of the Pantograph Energy Based on Material Symmetries}

We consider in the sequel the four basic pantographic structures pictured in Fig. 2, corresponding in general to a set of two non equivalent non orthogonal extensible fibers (Fig. 2d). We aim at computing the effective energy density of these structures based on their symmetry properties, as reflected by the corresponding material symmetry groups.

The four structures can be characterized as follows

- The square structure is characterized by two mutually orthogonal fibers in the reference configuration endowed with identical properties (tensile, shear and flexural rigidities)

- The rectangular structure has two orthogonal fibers but with different material properties

- The rhombic structure is made of two non orthogonal fibers with the same mechanical properties

- The parallelogram structure is the more general one since the two fibers are both non orthogonal and have different mechanical properties.

The material symmetry groups of these four patterns have been exposed in [3]. Their action on the postulated form of the strain energy density of the equivalent continuum leads to restrict the number and the form of the arguments of this density. Since we consider linear pantographic structures undergoing small perturbations, this entails we expect a decoupling of the first order and second order gradient contributions, so that we postulate an additive split of the total energy into

$$
\mathbf{W}(\mathbf{F}, \nabla \mathbf{F})=W_{1}(\mathbf{F})+W_{2}(\nabla \mathbf{F})
$$

with $W_{1}(\mathbf{F})$ the first order energy and $W_{2}(\nabla \mathbf{F})$ the second order energy contribution. The second order tensor $\mathbf{F}$ denotes the transformation gradient.

The $\nabla$ operator therein denotes the gradient with respect to the Lagrangian coordinate in a large strains framework. 



Figure 2. The four basic pantographic structures: a: square, b: rectangular, c: rhombic, d: parallelogram.

We adopt for convenience a basis made of the two fibers, with unit vectors in the reference configuration the two vectors $\mathbf{A}_{1}, \mathbf{A}_{2}$ in the direction of the two fibers. All kinematic tensors shall then be conveniently expressed in this basis.

For the first order gradient contribution, the energy $W_{1}(\mathbf{F})$ depends on the independent invariant $C_{11}, C_{22}$ and $C_{12}$, which are the components of the right Cauchy-Green tensor $\mathbf{C}=\mathbf{F}^{T} \cdot \mathbf{F}$. The following dependencies of $W_{1}(\mathbf{F})$ has been obtained based on an extension of Richlewski theorem in [3], from symmetry considerations (the material symmetry group associated to each pattern leads to restrictions on the arguments of the strain energy density)

- For the square symmetry: the material symmetry group includes the two planar rotations $R_{\pi / 2}=\left(\begin{array}{rr}0 & -1 \\ 1 & 0\end{array}\right)$ and $R_{1}=\left(\begin{array}{rr}-1 & 0 \\ 0 & 1\end{array}\right)$, thus leading to $W_{1}(\mathbf{F})=W_{1}\left(C_{11}+C_{22}, C_{11} C_{22}, C_{12}^{2}\right)$ 
- For the rectangular symmetry : the material symmetry group includes the two planar rotations $R_{1}=\left(\begin{array}{rr}-1 & 0 \\ 0 & 1\end{array}\right)$ and $R_{2}=\left(\begin{array}{rr}1 & 0 \\ 0 & -1\end{array}\right)$, thus leading to $W_{1}(\mathbf{F})=W_{1}\left(C_{11}, C_{22}, C_{12}^{2}\right)$

- For the rhombic symmetry: the material symmetry group includes the two planar rotations $R_{3}=\left(\begin{array}{rr}\cos \delta & \sin \delta \\ \sin \delta & -\cos \delta\end{array}\right)$ and $R_{4}=\left(\begin{array}{rr}-\cos \delta & -\sin \delta \\ -\sin \delta & \cos \delta\end{array}\right)$, with $\delta=2 \beta$, thus leading to $W_{1}(\mathbf{F})=W_{1}\left(C_{11}+C_{22}, C_{11} C_{22}, C_{12}\right)$

- For the parallelogram symmetry: the symmetry group describing the anisotropy of this network is $H_{p r}=\left\{\mathbf{I}=\left(\begin{array}{ll}1 & 0 \\ 0 & 1\end{array}\right),-\mathbf{I}=\left(\begin{array}{cc}-1 & 0 \\ 0 & -1\end{array}\right)\right\}$, thus leading to $W_{1}(\mathbf{F})=W_{1}\left(C_{11}, C_{22}, C_{12}\right)$.

For the second order gradient contribution, a similar argumentation leads to a second order energy $W_{2}(\nabla \mathbf{F})$ depending upon the following polynomial invariants

- For the square symmetry, the invariants are $\kappa_{1}^{2} \kappa_{2}^{2}, \kappa_{1}^{2}+\kappa_{2}^{2}, \sin ^{2} \gamma$

- For the rectangular symmetry, the invariants are $\kappa_{1}^{2}, \kappa_{2}^{2}, \sin ^{2} \gamma$

- For the rhombic symmetry, the invariants are $\kappa_{1}^{2} \kappa_{2}^{2}, \kappa_{1}^{2}+\kappa_{2}^{2}, \sin \gamma$

- For the parallelogram symmetry, the invariants are $\kappa_{1}^{2}, \kappa_{2}^{2}, \sin \gamma$.

The scalar coefficients $\gamma$ and $\kappa$ are respectively the shear angle and the curvature, defined as

$$
\kappa_{1}^{2}=\mathbf{a}_{1,1} \cdot \mathbf{a}_{1,1}, \quad \kappa_{2}^{2}=\mathbf{a}_{2,2} \cdot \mathbf{a}_{2,2}, \quad \sin \gamma=\mathbf{a}_{1} \cdot \mathbf{a}_{2} .
$$

Regarding notations, the comma denotes the derivative.

The list of arguments of the energy density further show that the sign of the shear angle matters for both the rhombic and parallelogram structures.

We shall next elaborate the kinematics of the homogenized pantograph considering the more general parallelogram structure, as pictured in Fig. 3.

The directors along the two deformed arcs are obtained by the push-forward of the two unit fiber vectors, defined as the unit directors along the two fibers, vectors $\mathbf{A}_{1}$ and $\mathbf{A}_{2}$, in the reference configuration

$$
\mathbf{a}_{1}=\mathbf{F} \cdot \mathbf{A}_{1}, \quad \mathbf{a}_{2}=\mathbf{F} \cdot \mathbf{A}_{2}
$$


$\Omega_{0}$
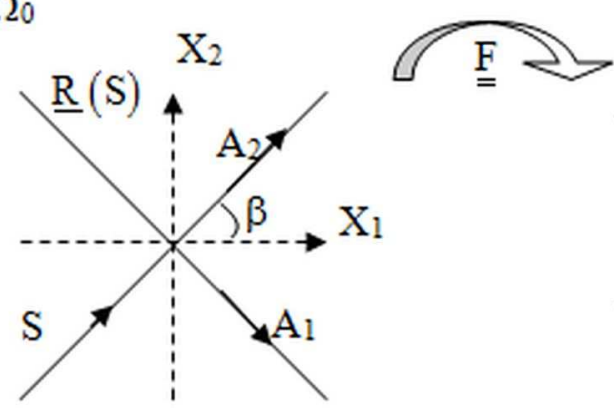

$\Omega_{\mathrm{t}}$

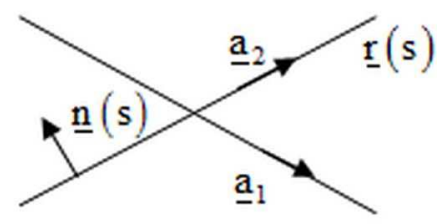

Figure 3. Reference Cartesian coordinates and parameterization of the parallelogram pantographic structure in the reference and actual configurations.

From the displacement vector expressed in the local basis attached to each fiber, viz

$$
\mathbf{u}=\xi \mathbf{e}^{b}+\eta \mathbf{e}^{b \perp}
$$

with $\mathbf{e}^{b}$ the unit director along each beam and $\mathbf{e}^{b \perp}$ the normal unit vector. It follows the displacement derivative with respect to the referential curvilinear coordinate denoted $\mathrm{S}$ is

$$
\frac{\mathrm{d} \mathbf{u}}{\mathrm{d} S}=\frac{\mathrm{d} \xi}{\mathrm{d} S} \mathbf{e}^{\mathbf{b}}+\frac{\mathrm{d} \eta}{\mathrm{d} S} \mathbf{e}^{\mathbf{b} \perp} .
$$

By considering both fibers this entails also the expression for the transformation gradient

$$
\mathbf{F}=\left(\begin{array}{cc}
1+\frac{\mathrm{d} \xi_{1}}{\mathrm{~d} S} & \frac{\mathrm{d} \eta_{1}}{\mathrm{~d} S} \\
\frac{\mathrm{d} \eta_{2}}{\mathrm{~d} S} & 1+\frac{\mathrm{d} \xi_{2}}{\mathrm{~d} S}
\end{array}\right)
$$

After elementary computations this leads to the expression of the three independent components of the right Cauchy-Green tensor in the referential basis made of the two fiber directors $\mathbf{A}_{1}, \mathbf{A}_{2}$

$$
C_{11}=2 \xi_{1, S}+1, \quad C_{22}=2 \xi_{2, S}+1, \quad C_{12}=\eta_{1, S}+\eta_{2, S} .
$$

Based on previous classification this leads to the following form of the first order gradient energy of parallelogram pantographic structures

$$
W_{1}(\mathbf{F})=W_{1}\left(2 \xi_{1, S}+1,2 \xi_{2, S}+1, \eta_{1, S}+\eta_{2, S}\right) .
$$


The second order gradient energy of parallelogram structures can be written versus the square of the two principal curvatures and the sine of the shear angle $\gamma$ [3]

$$
W_{2}(\nabla \mathbf{F})=W_{2}\left(\kappa_{1}^{2}, \kappa_{2}^{2}, \sin \gamma\right)
$$

The second order energy density splits into a contribution due to transverse shear and another contribution reflecting second order extensional effects, which we next evaluate separately.

After lengthy development (see Appendix 1), the following closed form expression of the second order energy density is obtained

$$
\begin{aligned}
& W_{2}(\nabla \mathbf{F}) \\
& \quad=W_{2}\left(\left(\xi_{1, S_{1} S_{1}}\right)^{2}+\left(\eta_{2, S_{1} S_{1}}\right)^{2},\left(\xi_{2, S_{2} S_{2}}\right)^{2}+\left(\eta_{1, S_{2} S_{2}}\right)^{2}, \eta_{1, S}+\eta_{2, S}\right) .
\end{aligned}
$$

In order to identify the nature of the form of $W_{1}(\mathbf{F})$ and $W_{2}(\nabla \mathbf{F})$ versus their arguments, we next compute both energies in closed form for all patterns.

\section{Analytical Expression of the Energy for Extensible Beams}

Extending the work done in [13] for the square structure with inextensible beams (corresponding to pantographic structures), we evaluate the strain energy of a pantographic lattice with an arbitrary initial configuration, Figure 4, made of extensible beams. The general expression of the internal deformation energy associated to a beam element $(i-j, i+1-j+1)$, is written as [13]

$$
\begin{aligned}
& W=\frac{1}{2} \frac{E S}{L}\left(\xi_{i+1}^{j+1}-\xi_{i}^{j}\right)^{2}+\frac{1}{2} \frac{12 E I}{L^{3}}\left(\left(\eta_{i+1}^{j+1}-\eta_{i}^{j}\right)-\frac{L}{2}\left(\theta_{i+1}^{j+1}+\theta_{i}^{j}\right)\right)^{2} \\
& +\frac{1}{2} \frac{E I}{L}\left(\theta_{i+1}^{j+1}-\theta_{i}^{j}\right)^{2} .
\end{aligned}
$$

This energy is made of three terms representing the different forms of strain energy, namely extensional, flexural and shear contributions.

We have introduced therein the extensional rigidity $K_{l}=E S / L_{b}$, the flexural rigidity $K_{f}=E I / L_{b}$, the shear rigidity $K_{c}=12 E I / L_{b}^{3}$ and $L_{b}=a$ is the beam length.

Both beams in Fig. 4 have different shear rigidities, denoted $K_{c_{1}}$ and $K_{c_{2}}$, different flexural rigidities $K_{f_{1}}$ and $K_{f_{2}}$ and distinct extensional rigidities $K_{l_{1}}$ and $K_{l_{2}}$. The 


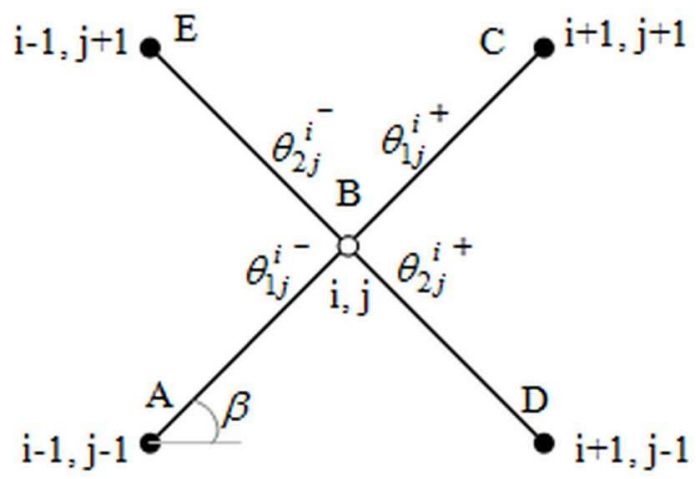

Figure 4. Unit cell of the pantograph structure. The link at node (i,j) is a pivot [13].

strain energy of the parallelogram pantographic structure writes as follows

$$
\begin{aligned}
& W=\frac{1}{2} K_{l_{1}}\left[\left(\xi_{1 j+1}^{i+1}-\xi_{1 j}^{i}\right)^{2}+\left(\xi_{1 j}^{i}-\xi_{1 j-1}^{i-1}\right)^{2}\right] \\
& +\frac{1}{2} K_{l_{2}}\left[\left(\xi_{2 j-1}^{i+1}-\xi_{2 j}^{i}\right)^{2}+\left(\xi_{2 j}^{i}-\xi_{2 j+1}^{i-1}\right)^{2}\right] \\
& +\frac{1}{2} K_{f_{1}}\left[\left(\theta_{1 j+1}^{i+1}-\theta_{1 j}^{i^{+}}\right)^{2}+\left(\theta_{1 j}^{i^{-}}-\theta_{1 j-1}^{i-1}\right)^{2}\right] \\
& +\frac{1}{2} K_{f_{2}}\left[\left(\theta_{2 j-1}^{i+1}-\theta_{2 j}^{i^{+}}\right)^{2}+\left(\theta_{2 j}^{i^{-}}-\theta_{2 j+1}^{i-1}\right)^{2}\right] \\
& +\frac{1}{2} K_{c_{1}}\left[\left(\left(\eta_{1 j+1}^{i+1}-\eta_{1 j}^{i}\right)-\frac{a}{2}\left(\theta_{1 j+1}^{i+1}+\theta_{1 j}^{i^{+}}\right)\right)^{2}\right. \\
& \left.+\left(\left(\eta_{1 j}^{i}-\eta_{1-1}^{i-1}\right)-\frac{a}{2}\left(\theta_{1 j}^{i^{-}}+\theta_{1 j-1}^{i-1}\right)\right)^{2}\right] \\
& +\frac{1}{2} K_{c_{2}}\left[\left(\left(\eta_{2 j-1}^{i+1}-\eta_{2 j}^{i}\right)-\frac{a}{2}\left(\theta_{2 j-1}^{i+1}+\theta_{2 j}^{i^{+}}\right)\right)^{2}\right. \\
& \left.\left(\left(\eta_{2 j}^{i}-\eta_{2}^{i-1}\right)-\frac{a}{2}\left(\theta_{2 j}^{i^{-}}+\theta_{2 j+1}^{i-1}\right)\right)^{2}\right] .
\end{aligned}
$$

The indices 1 and 2 refer respectively to the first and second beam. Using two different finite difference schemes further delivers the equalities

$$
\begin{aligned}
& \left(\xi_{j+1}^{i+1}-\xi_{j}^{i}\right)=\left.a \frac{\mathrm{d} \xi}{\mathrm{d} s}\right|_{j} ^{i}+\left.\frac{a^{2}}{2} \frac{\mathrm{d}^{2} \xi}{\mathrm{d} s^{2}}\right|_{j} ^{i} \\
& \left(\xi_{j}^{i}-\xi_{j-1}^{i-1}\right)=\left.a \frac{\mathrm{d} \xi}{\mathrm{d} s}\right|_{j} ^{i}-\left.\frac{a^{2}}{2} \frac{\mathrm{d}^{2} \xi}{\mathrm{d} s^{2}}\right|_{j} ^{i}
\end{aligned}
$$




$$
\begin{aligned}
& \left(\eta_{j+1}^{i+1}-\eta_{j}^{i}\right)=\left.a \frac{\mathrm{d} \eta}{\mathrm{d} s}\right|_{j} ^{i}+\left.\frac{a^{2}}{2} \frac{\mathrm{d}^{2} \eta}{\mathrm{d} s^{2}}\right|_{j} ^{i} \\
& \left(\eta_{j}^{i}-\eta_{j-1}^{i-1}\right)=\left.a \frac{\mathrm{d} \eta}{\mathrm{d} s}\right|_{j} ^{i}-\left.\frac{a^{2}}{2} \frac{\mathrm{d}^{2} \eta}{\mathrm{d} s^{2}}\right|_{j} ^{i}
\end{aligned}
$$

with s therein being the curvilinear abscissa along the beam.

According to Bernoulli model and the continuity of the angular variation of the beam at the central node, we can formulate for each beam the following continuity (two first equalities) and kinematic coupling conditions (last equality)

$$
\theta_{j}^{i^{+}}=\theta_{j}^{i^{-}}=\theta_{j}^{i}=\left.\frac{\mathrm{d} \eta}{\mathrm{d} s}\right|_{j} ^{i}
$$

The last equality means that the normal to any section of the beam remains normal during the motion.

After insertion of relations (4) through (7) into (3), the general energy expression of the parallelogram pantographic structure is obtained

$$
\begin{aligned}
W= & K_{l_{1}}\left(\left(\left.a \frac{\mathrm{d} \xi_{1}}{\mathrm{~d} s}\right|_{j} ^{i}\right)^{2}+\left(\left.\frac{a^{2}}{2} \frac{\mathrm{d}^{2} \xi_{1}}{\mathrm{~d} s^{2}}\right|_{j} ^{i}\right)^{2}\right) \\
& +K_{l_{2}}\left(\left(\left.a \frac{\mathrm{d} \xi_{2}}{\mathrm{~d} s}\right|_{j} ^{i}\right)^{2}+\left(\left.\frac{a^{2}}{2} \frac{\mathrm{d}^{2} \xi_{2}}{\mathrm{~d} s^{2}}\right|_{j} ^{i}\right)^{2}\right) \\
& +\frac{a^{4}}{4} K_{c_{1}}\left(\left.\frac{\mathrm{d}^{2} \eta_{1}}{\mathrm{~d} s^{2}}\right|_{j} ^{i}\right)^{2}+\frac{a^{4}}{4} K_{c_{2}}\left(\left.\frac{\mathrm{d}^{2} \eta_{2}}{\mathrm{~d} s^{2}}\right|_{j} ^{i}\right)^{2} .
\end{aligned}
$$

The energy is here expressed using the curvilinear coordinates associated to each fiber. We are now in a position to identify the specific form of the energy written in Section 2 (relation (1))

$$
\begin{aligned}
W= & K_{l_{1}}\left(a^{2}\left(\frac{C_{11}-1}{2}\right)^{2}+\frac{a^{2}}{4} \kappa_{1}^{2}\right)+K_{l_{2}}\left(a^{2}\left(\frac{C_{22}-1}{2}\right)^{2}+\frac{a^{2}}{4} \kappa_{2}^{2}\right) \\
& +\frac{a^{4}}{4}\left(\left(K_{c_{1}}-K_{l_{2}}\right)\left(\left.\frac{\mathrm{d}^{2} \eta_{1}}{\mathrm{~d} s^{2}}\right|_{j} ^{i}\right)^{2}+\left(K_{c_{2}}-K_{l_{1}}\right)\left(\left.\frac{\mathrm{d}^{2} \eta_{2}}{\mathrm{~d} s^{2}}\right|_{j} ^{i}\right)^{2}\right)
\end{aligned}
$$




$$
\begin{aligned}
= & a^{2}\left(K_{l_{1}}\left(\frac{C_{11}-1}{2}\right)^{2}+K_{l_{2}}\left(\frac{C_{22}-1}{2}\right)^{2}\right) \\
& +\frac{a^{4}}{4}\left(K_{l_{1}} \kappa_{1}^{2}+K_{l_{2}} \kappa_{2}^{2}+\left(K_{c_{1}}-K_{l_{2}}\right)\left(\left.\frac{\mathrm{d}^{2} \eta_{1}}{\mathrm{~d} s^{2}}\right|_{j} ^{i}\right)^{2}\right. \\
& \left.+\left(K_{c_{2}}-K_{l_{1}}\right)\left(\left.\frac{\mathrm{d}^{2} \eta_{2}}{\mathrm{~d} s^{2}}\right|_{j} ^{i}\right)^{2}\right) .
\end{aligned}
$$

This expression highlights that the energy of the parallelogram structure involves the components of the right Cauchy-Green tensor $C_{11}, C_{22}$, which define the first order energy

$$
W_{1}=K_{l_{1}} a^{2}\left(\frac{C_{11}-1}{2}\right)^{2}+K_{l_{2}} a^{2}\left(\frac{C_{22}-1}{2}\right)^{2}
$$

The remaining contribution in the right-hand side of equality (2) leads to the identification of the second order energy

$$
W_{2}=\frac{a^{4}}{4}\left(K_{l_{1}} \kappa_{1}^{2}+K_{l_{2}} \kappa_{2}^{2}+\left(K_{c_{1}}-K_{l_{2}}\right)\left(\left.\frac{\mathrm{d}^{2} \eta_{1}}{\mathrm{~d} s^{2}}\right|_{j} ^{i}\right)^{2}+\left(K_{c_{2}}-K_{l_{1}}\right)\left(\left.\frac{\mathrm{d}^{2} \eta_{2}}{\mathrm{~d} s^{2}}\right|_{j} ^{i}\right)^{2}\right)
$$

This expression shows that in addition to the squares of the two principal curvatures, the second order energy involves the two second order transverse shears $\frac{\mathrm{d}^{2} \eta_{1}}{\mathrm{~d} s^{2}}$ and $\frac{\mathrm{d}^{2} \eta_{2}}{\mathrm{~d} s^{2}}$. These two terms are in reality, accounting for Euler-Bernoulli assumption, the derivative of the rotation field of each beam with respect to the corresponding curvilinear coordinate.

In comparison to the general expression of $W_{2}$ obtained in the Appendix, this result also shows the absence of first order transverse shear, coefficient $C_{12}$.

The transition is next made from the local basis (attached to the beam) to the Cartesian basis: the local components $\xi_{1 j}^{i}, \xi_{2 j}^{i}, \eta_{1}{ }_{j}^{i}$ and $\eta_{2}{ }_{j}^{i}$ project along the two Cartesian components of the displacement vector $\left[\begin{array}{l}u \\ v\end{array}\right]$. After development of equation (9), one obtains the general energy expression for an arbitrary configuration in the 
Cartesian basis (for more details, see Appendix 2 and [13])

$$
\begin{aligned}
W= & \frac{a^{4}}{2} \cos \beta \sin \beta\left(K_{l_{1}}-K_{l_{2}}-K_{c_{1}}+K_{c_{2}}\right)\left(\frac{\mathrm{d}^{2} u}{\mathrm{~d} x^{2}}+\frac{\mathrm{d}^{2} u}{\mathrm{~d} y^{2}}\right)\left(\frac{\mathrm{d}^{2} v}{\mathrm{~d} x^{2}}+\frac{\mathrm{d}^{2} v}{\mathrm{~d} y^{2}}\right) \\
& +\frac{a^{4}}{4}\left(\frac{\mathrm{d}^{2} u}{\mathrm{~d} x^{2}}+\frac{\mathrm{d}^{2} u}{\mathrm{~d} y^{2}}\right)^{2}\left(\sin \beta^{2}\left(K_{c_{1}}+K_{l_{2}}\right)+\cos \beta^{2}\left(K_{c_{2}}+K_{l_{1}}\right)\right) \\
& +\frac{a^{4}}{4}\left(\frac{\mathrm{d}^{2} v}{\mathrm{~d} x^{2}}+\frac{\mathrm{d}^{2} v}{\mathrm{~d} y^{2}}\right)^{2}\left(\sin \beta^{2}\left(K_{c_{2}}+K_{l_{1}}\right)+\cos \beta^{2}\left(K_{c_{1}}+K_{l_{2}}\right)\right) \\
& +a^{2}\left(\frac{\mathrm{d} v}{\mathrm{~d} x}+\frac{\mathrm{d} v}{\mathrm{~d} y}\right)^{2}\left(\sin \beta^{2} K_{l_{1}}+\cos \beta^{2} K_{l_{2}}\right) \\
& +a^{2}\left(\frac{\mathrm{d} u}{\mathrm{~d} x}+\frac{\mathrm{d} u}{\mathrm{~d} y}\right)^{2}\left(\sin \beta^{2} K_{l_{2}}+\cos \beta^{2} K_{l_{1}}\right) .
\end{aligned}
$$

Note that the energy density $W / S$ can be evaluated by dividing $W$ by the area $S=4 a^{2} \sin \beta \cos \beta$. The three other patterns (the rhombic, the square and the rectangular structures) are specific cases of the more general parallelogram pattern (Fig. 2), so that it is straightforward to obtain their corresponding energy.

- For a square structure, it holds $\beta=\pi / 4, K_{l_{1}}=K_{l_{2}}$ and $K_{c_{1}}=K_{c_{2}}$, thus previous expression (10) of $W$ becomes

$$
\begin{aligned}
W= & a^{2} K_{l}\left(\left(\frac{\mathrm{d} u}{\mathrm{~d} x}+\frac{\mathrm{d} u}{\mathrm{~d} y}\right)^{2}+\left(\frac{\mathrm{d} v}{\mathrm{~d} x}+\frac{\mathrm{d} v}{\mathrm{~d} y}\right)^{2}\right) \\
& +\frac{a^{4}}{4}\left(K_{l}+K_{c}\right)\left(\left(\frac{\mathrm{d}^{2} u}{\mathrm{~d} x^{2}}+\frac{\mathrm{d}^{2} u}{\mathrm{~d} y^{2}}\right)^{2}+\left(\frac{\mathrm{d}^{2} v}{\mathrm{~d} x^{2}}+\frac{\mathrm{d}^{2} v}{\mathrm{~d} y^{2}}\right)^{2}\right) .
\end{aligned}
$$

This entails the energy density by dividing by the area of the elementary unit cell, that is $S=(\sqrt{2} a)^{2}$, viz

$$
\begin{aligned}
W / S= & \frac{1}{2} K_{l}\left(\left(\frac{\mathrm{d} u}{\mathrm{~d} x}+\frac{\mathrm{d} u}{\mathrm{~d} y}\right)^{2}+\left(\frac{\mathrm{d} v}{\mathrm{~d} x}+\frac{\mathrm{d} v}{\mathrm{~d} y}\right)^{2}\right) \\
& +\frac{a^{2}}{8}\left(K_{l}+K_{c}\right)\left(\left(\frac{\mathrm{d}^{2} u}{\mathrm{~d} x^{2}}+\frac{\mathrm{d}^{2} u}{\mathrm{~d} y^{2}}\right)^{2}+\left(\frac{\mathrm{d}^{2} v}{\mathrm{~d} x^{2}}+\frac{\mathrm{d}^{2} v}{\mathrm{~d} y^{2}}\right)^{2}\right)
\end{aligned}
$$


- For a rectangular structure, it holds $\beta=\pi / 4, K_{l_{1}} \neq K_{l_{2}}$ and $K_{c_{1}} \neq K_{c_{2}}$, thus previous expression (10) becomes

$$
\begin{aligned}
W= & \frac{a^{4}}{4}\left(K_{l_{1}}-K_{l_{2}}-K_{c_{1}}+K_{c_{2}}\right)\left(\frac{\mathrm{d}^{2} u}{\mathrm{~d} x^{2}}+\frac{\mathrm{d}^{2} u}{\mathrm{~d} y^{2}}\right)\left(\frac{\mathrm{d}^{2} v}{\mathrm{~d} x^{2}}+\frac{\mathrm{d}^{2} v}{\mathrm{~d} y^{2}}\right) \\
& +\frac{a^{4}}{8}\left(K_{c_{1}}+K_{c_{2}}+K_{l_{2}}+K_{l_{1}}\right)\left(\left(\frac{\mathrm{d}^{2} u}{\mathrm{~d} x^{2}}+\frac{\mathrm{d}^{2} u}{\mathrm{~d} y^{2}}\right)^{2}+\left(\frac{\mathrm{d}^{2} v}{\mathrm{~d} x^{2}}+\frac{\mathrm{d}^{2} v}{\mathrm{~d} y^{2}}\right)^{2}\right) \\
& +\frac{a^{2}}{2}\left(K_{l_{1}}+K_{l_{2}}\right)\left(\left(\frac{\mathrm{d} u}{\mathrm{~d} x}+\frac{\mathrm{d} u}{\mathrm{~d} y}\right)^{2}+\left(\frac{\mathrm{d} v}{\mathrm{~d} x}+\frac{\mathrm{d} v}{\mathrm{~d} y}\right)^{2}\right) \\
& +a^{2}\left(K_{l_{1}}-K_{l_{2}}\right)\left(\frac{\mathrm{d} u}{\mathrm{~d} x}+\frac{\mathrm{d} u}{\mathrm{~d} y}\right)\left(\frac{\mathrm{d} v}{\mathrm{~d} x}+\frac{\mathrm{d} v}{\mathrm{~d} y}\right) .
\end{aligned}
$$

This entails the energy density

$$
\begin{aligned}
W / S= & \frac{a^{2}}{8}\left(K_{l_{1}}-K_{l_{2}}-K_{c_{1}}+K_{c_{2}}\right)\left(\frac{\mathrm{d}^{2} u}{\mathrm{~d} x^{2}}+\frac{\mathrm{d}^{2} u}{\mathrm{~d} y^{2}}\right)\left(\frac{\mathrm{d}^{2} v}{\mathrm{~d} x^{2}}+\frac{\mathrm{d}^{2} v}{\mathrm{~d} y^{2}}\right) \\
& +\frac{a^{2}}{16}\left(K_{c_{1}}+K_{c_{2}}+K_{l_{2}}+K_{l_{1}}\right)\left(\left(\frac{\mathrm{d}^{2} u}{\mathrm{~d} x^{2}}+\frac{\mathrm{d}^{2} u}{\mathrm{~d} y^{2}}\right)^{2}+\left(\frac{\mathrm{d}^{2} v}{\mathrm{~d} x^{2}}+\frac{\mathrm{d}^{2} v}{\mathrm{~d} y^{2}}\right)^{2}\right) \\
& +\frac{1}{4}\left(K_{l_{1}}+K_{l_{2}}\right)\left(\left(\frac{\mathrm{d} u}{\mathrm{~d} x}+\frac{\mathrm{d} u}{\mathrm{~d} y}\right)^{2}+\left(\frac{\mathrm{d} v}{\mathrm{~d} x}+\frac{\mathrm{d} v}{\mathrm{~d} y}\right)^{2}\right) \\
& +\frac{1}{2}\left(K_{l_{1}}-K_{l_{2}}\right)\left(\frac{\mathrm{d} u}{\mathrm{~d} x}+\frac{\mathrm{d} u}{\mathrm{~d} y}\right)\left(\frac{\mathrm{d} v}{\mathrm{~d} x}+\frac{\mathrm{d} v}{\mathrm{~d} y}\right) .
\end{aligned}
$$

- For a rhombic structure, with $\beta=\pi / 6, K_{l_{1}}=K_{l_{2}}$ and $K_{c_{1}}=K_{c_{2}}$, previous expression (10) becomes

$$
\begin{aligned}
W= & \frac{a^{4}}{4}\left(K_{c}+K_{l}\right)\left(\left(\frac{\mathrm{d}^{2} u}{\mathrm{~d} x^{2}}+\frac{\mathrm{d}^{2} u}{\mathrm{~d} y^{2}}\right)^{2}+\left(\frac{\mathrm{d}^{2} v}{\mathrm{~d} x^{2}}+\frac{\mathrm{d}^{2} v}{\mathrm{~d} y^{2}}\right)^{2}\right) \\
& +a^{2} K_{l}\left(\left(\frac{\mathrm{d} u}{\mathrm{~d} x}+\frac{\mathrm{d} u}{\mathrm{~d} y}\right)^{2}+\left(\frac{\mathrm{d} v}{\mathrm{~d} x}+\frac{\mathrm{d} v}{\mathrm{~d} y}\right)^{2}\right) .
\end{aligned}
$$

This entails the energy density

$$
\begin{aligned}
W / S= & \frac{a^{2}}{12}\left(K_{c}+K_{l}\right)\left(\left(\frac{\mathrm{d}^{2} u}{\mathrm{~d} x^{2}}+\frac{\mathrm{d}^{2} u}{\mathrm{~d} y^{2}}\right)^{2}+\left(\frac{\mathrm{d}^{2} v}{\mathrm{~d} x^{2}}+\frac{\mathrm{d}^{2} v}{\mathrm{~d} y^{2}}\right)^{2}\right) \\
& +\frac{1}{3} K_{l}\left(\left(\frac{\mathrm{d} u}{\mathrm{~d} x}+\frac{\mathrm{d} u}{\mathrm{~d} y}\right)^{2}+\left(\frac{\mathrm{d} v}{\mathrm{~d} x}+\frac{\mathrm{d} v}{\mathrm{~d} y}\right)^{2}\right) .
\end{aligned}
$$


The Cauchy first and second order stress tensors are obtained for the parallelogram pattern as

$$
\begin{aligned}
& \sigma_{x x}=\frac{\mathrm{d} W / S}{\mathrm{~d} u / \mathrm{d} x}=\frac{1}{2 \sin \beta \cos \beta}\left(\cos \beta \sin \beta\left(K_{l_{1}}-K_{l_{2}}\right)\left(\frac{\mathrm{d} v}{\mathrm{~d} x}+\frac{\mathrm{d} v}{\mathrm{~d} y}\right)\right. \\
& \left.+\left(\sin \beta^{2} K_{l_{2}}+\cos \beta^{2} K_{l_{1}}\right)\left(\frac{\mathrm{d} u}{\mathrm{~d} x}+\frac{\mathrm{d} u}{\mathrm{~d} y}\right)\right)=\sigma_{x y}=\frac{\mathrm{d} W / S}{\mathrm{~d} u / \mathrm{d} y} \\
& \sigma_{y y}=\frac{\mathrm{d} W / S}{\mathrm{~d} v / \mathrm{d} y}=\frac{1}{2 \sin \beta \cos \beta}\left(\cos \beta \sin \beta\left(K_{l_{1}}-K_{l_{2}}\right)\left(\frac{\mathrm{d} u}{\mathrm{~d} x}+\frac{\mathrm{d} u}{\mathrm{~d} y}\right)\right. \\
& \left.+\left(\sin \beta^{2} K_{l_{1}}+\cos \beta^{2} K_{l_{2}}\right)\left(\frac{\mathrm{d} v}{\mathrm{~d} x}+\frac{\mathrm{d} v}{\mathrm{~d} y}\right)\right)=\sigma_{y x}=\frac{\mathrm{d} W / S}{\mathrm{~d} v / \mathrm{d} x} \\
& S_{x x x}=\frac{\mathrm{d} W / S}{\mathrm{~d}^{2} u / \mathrm{d} x^{2}} \\
& =\frac{a^{2}}{8 \sin \beta \cos \beta}\left(\cos \beta \sin \beta\left(K_{l_{1}}-K_{l_{2}}-K_{c_{1}}+K_{c_{2}}\right)\left(\frac{\mathrm{d}^{2} v}{\mathrm{~d} x^{2}}+\frac{\mathrm{d}^{2} v}{\mathrm{~d} y^{2}}\right)\right. \\
& \left.+\left(\sin \beta^{2}\left(K_{c_{1}}+K_{l_{2}}\right)+\cos \beta^{2}\left(K_{c_{2}}+K_{l_{1}}\right)\right)\left(\frac{\mathrm{d}^{2} u}{\mathrm{~d} x^{2}}+\frac{\mathrm{d}^{2} u}{\mathrm{~d} y^{2}}\right)\right) \\
& =S_{x y y}=\frac{\mathrm{d} W / S}{\mathrm{~d}^{2} u / \mathrm{d} y^{2}} \\
& S_{y x x}=\frac{\mathrm{d} W / S}{\mathrm{~d}^{2} v / \mathrm{d} x^{2}} \\
& =\frac{a^{2}}{8 \sin \beta \cos \beta}\left(\cos \beta \sin \beta\left(K_{l_{1}}-K_{l_{2}}-K_{c_{1}}+K_{c_{2}}\right)\left(\frac{\mathrm{d}^{2} u}{\mathrm{~d} x^{2}}+\frac{\mathrm{d}^{2} u}{\mathrm{~d} y^{2}}\right)\right. \\
& \left.+\left(\sin \beta^{2}\left(K_{c_{2}}+K_{l_{1}}\right)+\cos \beta^{2}\left(K_{c_{1}}+K_{l_{2}}\right)\right)\left(\frac{\mathrm{d}^{2} v}{\mathrm{~d} x^{2}}+\frac{\mathrm{d}^{2} v}{\mathrm{~d} y^{2}}\right)\right) \\
& =S_{y y y}=\frac{\mathrm{d} W / S}{\mathrm{~d}^{2} v / \mathrm{d} y^{2}} .
\end{aligned}
$$

Both stress and hyperstress tensors satisfy the continuum static equilibrium equation

$$
\nabla \cdot(\sigma-\nabla \cdot(\mathbf{S}))+\mathbf{f}=\mathbf{0}
$$




\section{Conclusion: Discussion and Perspectives}

The mechanics of planar networks of extensible fibers has been analyzed, for which the general form of the mechanical energy has been derived, based on a classification of the studied patterns in terms of material symmetries. We considered a network made of two sets of non orthogonal and non equivalent fibers, called the parallelogram structure, with subcases coined the square, rectangular, and rhombic patterns. The arguments of the energy of these four patterns are obtained based on the material symmetry group of the considered structures. The energy of all four patterns is then obtained in closed form, thus leading to the identification of the coefficients associated to each of the obtained arguments. The fibers of the network are assumed to obey Bernoulli kinematics. A second order gradient continuum is obtained by a continualisation of the beam equations by adopting a second order finite difference scheme for the kinematic variables.

The Cauchy first order stress and the second order hyperstress tensors have been derived from the formulated energy density of the fiber network. The present work paves the way to structural computations at the macroscopic level relying on the obtained energy density of the equivalent continuum which has been substituted to the initially discrete network. The extension to large strains shall also deserve future investigations.

\section{Acknowledgements}

The authors would like to thank the support of the PICS 06518 between Tunisia and France, which made this collaboration between both Institutes possible.

\section{Appendix 1: Identification of the Arguments of the Second Order Gradient Energy}

The second order gradient energy of parallelogram pantographic structures undergoing small perturbations can be written as

$$
W_{2}(\nabla \mathbf{F})=W_{2}\left(\kappa_{1}^{2}, \kappa_{2}^{2}, \sin \gamma\right)=W_{2}\left(\mathbf{a}_{1,1} \cdot \mathbf{a}_{1,1}, \mathbf{a}_{2,2} \cdot \mathbf{a}_{2,2}, \mathbf{a}_{1} \cdot \mathbf{a}_{2}\right) .
$$

In the following, one calculates separately each of the three arguments of $W_{2}$

$$
\mathbf{a}_{1,1} \cdot \mathbf{a}_{1,1}=\frac{\mathrm{d} \mathbf{a}_{1}}{\mathrm{~d} X_{1}} \cdot \frac{\mathrm{d} \mathbf{a}_{1}}{\mathrm{~d} X_{1}} .
$$

From Fig. 3, one can write $\frac{\mathrm{d} a_{1}}{\mathrm{~d} X_{1}}=\frac{\mathrm{d} a_{1}}{\mathrm{~d} S_{1}} \cdot \frac{\mathrm{d} S_{1}}{\mathrm{~d} X_{1}}=\frac{1}{\cos \beta} \frac{\mathrm{d} a_{1}}{\mathrm{~d} S_{1}}$ with $\mathbf{a}_{1}=\mathbf{F} . \mathbf{A}_{1}$ and $\mathbf{a}_{2}=\mathbf{F} . \mathbf{A}_{2}$. 
Hence, it holds

$$
\frac{\mathrm{d} \mathbf{a}_{1}}{\mathrm{~d} S_{1}}=\frac{\mathrm{d}}{\mathrm{d} S_{1}}\left(\mathbf{F} \cdot \mathbf{A}_{1}\right)=\frac{\mathrm{d} \mathbf{F}}{\mathrm{d} S_{1}} \cdot \mathbf{A}_{1}=M \cdot \mathbf{A}_{1}
$$

with the matrix

$$
M=\left(\begin{array}{cc}
\xi_{1, S_{1} S_{1}} & \eta_{1, S_{1} S_{1}} \\
\eta_{2, S_{1} S_{1}} & \xi_{2, S_{1} S_{1}}
\end{array}\right)
$$

Thus, one obtains

$$
\begin{aligned}
\mathbf{a}_{1,1} \cdot \mathbf{a}_{1,1}=\frac{1}{\cos ^{2} \beta} \frac{\mathrm{d} \mathbf{a}_{1}}{\mathrm{~d} S_{1}} \cdot \frac{\mathrm{d} \mathbf{a}_{1}}{\mathrm{~d} S_{1}}=\frac{1}{\cos ^{2} \beta} \mathbf{A}_{1} \cdot M^{T} \cdot M \cdot \mathbf{A}_{1} \\
=\left(\xi_{1, S_{1} S_{1}}\right)^{2}+\left(\eta_{2, S_{1} S_{1}}\right)^{2}
\end{aligned}
$$

with

$$
M^{T} \cdot M \approx\left(\begin{array}{cc}
\left(\xi_{1, S_{1} S_{1}}\right)^{2}+\left(\eta_{2, S_{1} S_{1}}\right)^{2} & 0 \\
0 & \left(\xi_{2, S_{1} S_{1}}\right)^{2}+\left(\eta_{1, S_{1} S_{1}}\right)^{2}
\end{array}\right) .
$$

The same development is done for $\mathbf{a}_{2,2} \cdot \mathbf{a}_{2,2}$, thus

$$
\begin{aligned}
\mathbf{a}_{2,2} \cdot \mathbf{a}_{2,2}=\frac{1}{\cos ^{2} \beta} \frac{\mathrm{d} \mathbf{a}_{2}}{\mathrm{~d} S_{2}} \cdot \frac{\mathrm{d} \mathbf{a}_{2}}{\mathrm{~d} S_{2}}=\frac{1}{\cos ^{2} \beta} \mathbf{A}_{2} \cdot N^{T} \cdot N \cdot \mathbf{A}_{2} \\
=\left(\xi_{2, S_{2} S_{2}}\right)^{2}+\left(\eta_{1, S_{2} S_{2}}\right)^{2} .
\end{aligned}
$$

Finally, $\mathbf{a}_{1} \cdot \mathbf{a}_{2}=\mathbf{C}_{12} \approx \eta_{1, S}+\eta_{2, S}$ with

$$
\mathbf{F}^{\mathbf{T}} \cdot \mathbf{F}=\left(\begin{array}{cc}
\left(1+\xi_{1, S}\right)^{2}+\left(\eta_{2, S}\right)^{2} & \eta_{2, S}+\eta_{1, S}+\eta_{1, S} \xi_{1, S}+\eta_{2, S} \xi_{2, S} \\
\eta_{2, S}+\eta_{1, S}+\eta_{1, S} \xi_{1, S}+\eta_{2, S} \xi_{2, S} & \left(1+\xi_{2, S}\right)^{2}+\left(\eta_{1, S}\right)^{2}
\end{array}\right) .
$$

Summarizing, we obtain the following general form of the second order energy density

$$
W_{2}(\nabla \mathbf{F})=W_{2}\left(\left(\xi_{1, S_{1} S_{1}}\right)^{2}+\left(\eta_{2, S_{1} S_{1}}\right)^{2},\left(\xi_{2, S_{2} S_{2}}\right)^{2}+\left(\eta_{1, S_{2} S_{2}}\right)^{2}, \eta_{1, S}+\eta_{2, S}\right)
$$

\section{Appendix 2: Transition From Curvilinear to Cartesian Coordinates}

It holds for the first beam with extremity $(i-1, j-1 ; i+1, j+1)$

$$
\left.\xi_{1}\right|_{j} ^{i}=\cos \beta u+\sin \beta v,\left.\quad \eta_{1}\right|_{j} ^{i}=-\sin \beta u+\cos \beta v .
$$

Similarly, one can write for the second beam $(i+1, j-1 ; i-1, j+1)$

$$
\left.\xi_{2}\right|_{j} ^{i}=-\sin \beta u+\cos \beta v,\left.\quad \eta_{2}\right|_{j} ^{i}=-\cos \beta u-\sin \beta v .
$$


This entails the first and second derivatives (for more details see [13]), with $\mathbf{s}$ represents the curvilinear coordinate along any beam element

$$
\begin{aligned}
& \left.\frac{\mathrm{d} \xi_{1}}{\mathrm{~d} s}\right|_{j} ^{i}=\left(\left.\frac{\mathrm{d} \xi_{1}}{\mathrm{~d} x}\right|_{j} ^{i}+\left.\frac{\mathrm{d} \xi_{1}}{\mathrm{~d} y}\right|_{j} ^{i}\right)=\cos \beta\left(\frac{\mathrm{d} u}{\mathrm{~d} x}+\frac{\mathrm{d} u}{\mathrm{~d} y}\right)+\sin \beta\left(\frac{\mathrm{d} v}{\mathrm{~d} x}+\frac{\mathrm{d} v}{\mathrm{~d} y}\right) \\
& \left.\frac{\mathrm{d} \eta_{1}}{\mathrm{~d} s}\right|_{j} ^{i}=\left(\left.\frac{\mathrm{d} \eta_{1}}{\mathrm{~d} x}\right|_{j} ^{i}+\left.\frac{\mathrm{d} \eta_{1}}{\mathrm{~d} y}\right|_{j} ^{i}\right)=\cos \beta\left(\frac{\mathrm{d} v}{\mathrm{~d} x}+\frac{\mathrm{d} v}{\mathrm{~d} y}\right)-\sin \beta\left(\frac{\mathrm{d} u}{\mathrm{~d} x}+\frac{\mathrm{d} u}{\mathrm{~d} y}\right) \\
& \left.\frac{\mathrm{d} \xi_{2}}{\mathrm{~d} s}\right|_{j} ^{i}=\left(\left.\frac{\mathrm{d} \xi_{2}}{\mathrm{~d} x}\right|_{j} ^{i}+\left.\frac{\mathrm{d} \xi_{2}}{\mathrm{~d} y}\right|_{j} ^{i}\right)=-\sin \beta\left(\frac{\mathrm{d} u}{\mathrm{~d} x}+\frac{\mathrm{d} u}{\mathrm{~d} y}\right)+\cos \beta\left(\frac{\mathrm{d} v}{\mathrm{~d} x}+\left.\frac{\mathrm{d} v}{\mathrm{~d} y}\right|_{j}=\left(\left.\frac{\mathrm{d} \eta_{2}}{\mathrm{~d} x}\right|_{j} ^{i}+\left.\frac{\mathrm{d} \eta_{2}}{\mathrm{~d} y}\right|_{j} ^{i}\right)=-\cos \beta\left(\frac{\mathrm{d} u}{\mathrm{~d} x}+\frac{\mathrm{d} u}{\mathrm{~d} y}\right)-\sin \beta\left(\frac{\mathrm{d} v}{\mathrm{~d} x}+\frac{\mathrm{d} v}{\mathrm{~d} y}\right)\right. \\
& \left.\frac{\mathrm{d}^{2} \xi_{1}}{\mathrm{~d} s^{2}}\right|_{j} ^{i}=\left(\left.\frac{\mathrm{d}^{2} \xi_{1}}{\mathrm{~d} x^{2}}\right|_{j} ^{i}+\left.\frac{\mathrm{d}^{2} \xi_{1}}{\mathrm{~d} y^{2}}\right|_{j} ^{i}\right)=\cos \beta\left(\frac{\mathrm{d}^{2} u}{\mathrm{~d} x^{2}}+\frac{\mathrm{d}^{2} u}{\mathrm{~d} y^{2}}\right)+\sin \beta\left(\frac{\mathrm{d}^{2} v}{\mathrm{~d} x^{2}}+\frac{\mathrm{d}^{2} v}{\mathrm{~d} y^{2}}\right) \\
& \left.\frac{\mathrm{d}^{2} \eta_{1}}{\mathrm{~d} s^{2}}\right|_{j} ^{i}=\left(\left.\frac{\mathrm{d}^{2} \eta_{1}}{\mathrm{~d} x^{2}}\right|_{j} ^{i}+\left.\frac{\mathrm{d}^{2} \eta_{1}}{\mathrm{~d} y^{2}}\right|_{j} ^{i}\right)=-\sin \beta\left(\frac{\mathrm{d}^{2} u}{\mathrm{~d} x^{2}}+\frac{\mathrm{d}^{2} u}{\mathrm{~d} y^{2}}\right)+\cos \beta\left(\frac{\mathrm{d}^{2} v}{\mathrm{~d} x^{2}}+\frac{\mathrm{d}^{2} v}{\mathrm{~d} y^{2}}\right) \\
& \left.\frac{\mathrm{d}^{2} \xi_{2}}{\mathrm{~d} s^{2}}\right|_{j} ^{i}=\left(\left.\frac{\mathrm{d}^{2} \xi_{2}}{\mathrm{~d} x^{2}}\right|_{j} ^{i}+\left.\frac{\mathrm{d}^{2} \xi_{2}}{\mathrm{~d} y^{2}}\right|_{j} ^{i}\right)=-\sin \beta\left(\frac{\mathrm{d}^{2} u}{\mathrm{~d} x^{2}}+\frac{\mathrm{d}^{2} u}{\mathrm{~d} y^{2}}\right)+\cos \beta\left(\frac{\mathrm{d}^{2} v}{\mathrm{~d} x^{2}}+\frac{\mathrm{d}^{2} v}{\mathrm{~d} y^{2}}\right) \\
& \left.\frac{\mathrm{d}^{2} \eta_{2}}{\mathrm{~d} s^{2}}\right|_{j} ^{i}=\left(\left.\frac{\mathrm{d}^{2} \eta_{2}}{\mathrm{~d} x^{2}}\right|_{j} ^{i}+\left.\frac{\mathrm{d}^{2} \eta_{2}}{\mathrm{~d} y^{2}}\right|_{j} ^{i}\right)=-\cos \beta\left(\frac{\mathrm{d}^{2} u}{\mathrm{~d} x^{2}}+\frac{\mathrm{d}^{2} u}{\mathrm{~d} y^{2}}\right)-\sin \beta\left(\frac{\mathrm{d}^{2} v}{\mathrm{~d} x^{2}}+\frac{\mathrm{d}^{2} v}{\mathrm{~d} y^{2}}\right) .
\end{aligned}
$$

Thus, after development, the energy (9) writes

$$
\begin{aligned}
W= & \frac{a^{4}}{2} \cos \beta \sin \beta\left(K_{l_{1}}-K_{l_{2}}-K_{c_{1}}+K_{c_{2}}\right)\left(\frac{\mathrm{d}^{2} u}{\mathrm{~d} x^{2}}+\frac{\mathrm{d}^{2} u}{\mathrm{~d} y^{2}}\right)\left(\frac{\mathrm{d}^{2} v}{\mathrm{~d} x^{2}}+\frac{\mathrm{d}^{2} v}{\mathrm{~d} y^{2}}\right) \\
& +\frac{a^{4}}{4}\left(\frac{\mathrm{d}^{2} u}{\mathrm{~d} x^{2}}+\frac{\mathrm{d}^{2} u}{\mathrm{~d} y^{2}}\right)^{2}\left(\sin \beta^{2}\left(K_{c_{1}}+K_{l_{2}}\right)+\cos \beta^{2}\left(K_{c_{2}}+K_{l_{1}}\right)\right) \\
& +\frac{a^{4}}{4}\left(\frac{\mathrm{d}^{2} v}{\mathrm{~d} x^{2}}+\frac{\mathrm{d}^{2} v}{\mathrm{~d} y^{2}}\right)^{2}\left(\sin \beta^{2}\left(K_{c_{2}}+K_{l_{1}}\right)+\cos \beta^{2}\left(K_{c_{1}}+K_{l_{2}}\right)\right) \\
& +a^{2}\left(\frac{\mathrm{d} v}{\mathrm{~d} x}+\frac{\mathrm{d} v}{\mathrm{~d} y}\right)^{2}\left(\sin \beta^{2} K_{l_{1}}+\cos \beta^{2} K_{l_{2}}\right) \\
& +a^{2}\left(\frac{\mathrm{d} u}{\mathrm{~d} x}+\frac{\mathrm{d} u}{\mathrm{~d} y}\right)^{2}\left(\sin \beta^{2} K_{l_{2}}+\cos \beta^{2} K_{l_{1}}\right) .
\end{aligned}
$$




\section{References}

[1] Bardenhagen S. and Triantafyllidis N., Derivation of Higher Order Gradient Continuum Theories in 2, 3-D Nonlinear Elasticity from Periodic Lattice Models, J. Mech. Phys. Solids 42 (1994) 111-139.

[2] Camar-Eddine M. and Seppecher P., Determination of the Closure of the Set of Elasticity Functionals, Arch. Rational Mech. Anal. 170 (2003) 211-245.

[3] Indelicato G., Mechanical Models for 2D Fiber Networks and Textiles, PhD Thesis, Torino 2008.

[4] dell'Isola F. and Steigmann D., A Two-Dimensional Gradient-Elasticity Theory for Woven Fabrics, J. Elast. 118 (2015) 113-125.

[5] Luo C. and Steigmann D., Bending and Twisting Effects in the ThreeDimensional Finite Deformations of an Inextensible Network, Chapter In: Advances in the Mechanics of Plates and Shells, vol. 88, Kluwer, New York 2002 pp.213-228.

[6] Mareno A. and Healey T., Global Continuation in Second-Gradient Nonlinear Elasticity, SIAM J. Math. Anal. 38 (2006) 103-115.

[7] McVeigh C., Vernerey F., Liu W. and Brinson L., Multiresolution Analysis for Material Design, Comput. Method. Appl. Mech. Eng. 195 (2006) 50535076.

[8] Mindlin R., Second Gradient of Strain and Surface Tension in Linear Elasticity, Int. J. Solids Struct. 1 (1965) 417-438.

[9] Pideri C. and Seppecher P., A Second Gradient Material Resulting from the Homogenization of a Heterogeneous Linear Elastic Medium, Continuum Mech. Thermodyn., Springer 9 (1997) 241-257.

[10] Pipkin A., Some Developments in the Theory of Inextensible Networks, Quart. Appl. Math. 38 (1980) 343-355.

[11] Pipkin A., Plane Traction Problems for Inextensible Networks, Quart. J. Mech. Appl. Math. 34 (1981) 415-429.

[12] Pipkin A., Inextensible Networks with Slack, Quart. Appl. Math. 40 (1982/1983) 63-71.

[13] Rahali Y., Giorgio I., Ganghoffer J. and dell'Isola F., Homogenization à la Piola Produces Second Gradient Continuum Models for Linear Pantographic Lattices, Inter. J. Eng. Sci. 97 (2015) 148-172.

[14] dos Reis F. and Ganghoffer J., Construction of Micropolar Continua from the Asymptotic Homogenization of Beam Lattices, Comput. Struct. 112-113 (2012) 354-363. 
[15] Rivlin R., Plane Strain of a Net Formed by Inextensible Cords, Chapter In Collected Papers of R. Rivlin, Springer, New York 1955, pp 511-534.

[16] Steigmann D. and dell'Isola F., Mechanical Response of Fabric Sheets to Three-Dimensional Bending, Twisting, and Stretching, Acta Mech. Sin. 31 (2015) 373-382.

[17] Trinh D., Janicke R., Auffray N., Diebels S. and Forest S., Evaluation of Generalized Continuum Substitution Models for Heterogeneous Materials, Inter. J. Mult. Comp. Eng. 10 (2011) 527-549.

[18] Wang W. and Pipkin A., Inextensible Networks with Bending Stiffness, Quart. J. Mech. Appl. Math. 39 (1986) 343-359.

[19] Wang W. and Pipkin A., Plane Deformations of Nets with Bending Stiffness, J. Acta Mech. 65 (1987) 263-279.

Received 01 November 2015

Yosra Rahali

MSSDT, ESSTT

Université de Tunis, Tunisie

E-mail address: rahali.yosra@gmail.com

Jean-François Ganghoffer

LEMTA, Université de Lorraine

Nancy, France

E-mail address: jean-francois.Ganghofferduniv-lorraine.fr

Frej Chaouachi

MSSDT, ESSTT

Université de Tunis, Tunisie

E-mail address: frej_ch@yahoo.fr

Ali Zghal

MSSDT, ESSTT

Université de Tunis, Tunisie

E-mail address: ali.zghal@gmail.com 